\title{
Structuralism, causation and explanation
}

\author{
Andreas Hüttemann 1
}

Published online: 14 June 2017

(c) Springer Science+Business Media B.V. 2017

Structuralism, conceived of as a metaphysical position in philosophy of science, is motivated by two different lines of thought. Firstly, an observation about theory change has been taken to indicate that what is preserved in theory change-and thus does not fall prey to pessimistic meta-induction-is the structure postulated and represented in the theories' mathematical equations rather than the entities postulated by the theories.

"There was an important element of continuity in the shift from Fresnel to Maxwell—and this was much more than a simple question of carrying over the successful empirical content into the new theory. At the same time it was rather less than a carrying over of the full theoretical content or full theoretical mechanisms (even in "approximate" form)... There was continuity or accumulation in the shift, but the continuity is one of form or structure, not of content." (Worrall 1989, p. 117)

For a scientific realist, it is argued, it is reasonable to be a realist about structure rather than about entities.

Secondly, structuralism is supported by the interpretation of some aspects of fundamental physical theories. Quantum mechanical entanglement and the phenomenon of so-called identical particles are difficult to reconcile with traditional metaphysical views about the identity of entities in terms of intrinsic properties (see Ladyman 2016 for a review).

Structuralism raises quite a number of questions, such as What is structure? and What is the difference between physical and mathematical structure? Among the controversial issues is furthermore the question what role causation plays within a structuralist account of reality. The papers of this special issue focus on the question of the role of causation and causal explanations vis-á-vis structuralism.

$凶$ Andreas Hüttemann

ahuettem@uni-koeln.de

1 Cologne, Germany 
Juha Saatsi explores to what extent the notion of causation helps to clarify the structuralist conception of structure. For this purpose he distinguishes various forms of structuralism: causal structuralism, ontic structural realism and invariance structuralism. Saatsi disputes the causal structuralists' claim that ties structure to the causal profile of the fundamental properties. Rather, fundamental laws and the properties that figure in the laws are non-causal. While causal structuralism is thus not a good candidate for spelling out structuralist intuition, in less fundamental domains of the sciences, Saatsi argue, the application of causal terminology might very well be reasonable.

Anjan Chakravartty diagnoses a prima facie tension when it comes to the application of causal terminology in a structuralist setting. Causation is usually conceived of as a relation between events which in turn involve objects. However, the status of objects in structuralism is somewhat precarious. Eliminative structuralism faces the challenge of explaining how relations by themselves can be causally efficacious, while noneliminative structuralism allows for entities that are dependent solely on the relations in which they stand - without having any intrinsic qualitative features. This, however, gives rise to the puzzle of what these entities are. Charkarvartty suggests that a view he dubs 'realist pragmatism' will overcome these problems.

Alexander Reutlinger's paper is concerned with the role of causation in Ladyman and Ross's version of structuralism as presented in Ladyman et al. (2007). Ladyman and Ross deny that there are fundamental causal facts but allow for emergent higher level causal facts in the special sciences. Reutlinger disputes that these latter facts are emergent in the technical sense Ladyman and Ross rely on. Reutlinger proceeds by closely analysing their positive argument, which relies on a non-reductive account of renormalization group explanations. He argues by contrast that these explanations can be understood as reductive explanations so that Ladyman and Ross's positive argument for higher level causal facts being emergent is undermined.

Mauro Dorato's paper returns to the issue of theory succession that originally motivated structuralism. Dorato observes that in some well-known cases of what Kuhn called scientific revolutions causal (dynamical) explanations are replaced by structural explanations, where structural explanation dissolve the need to appeal to causes or forces. This observation might be taken to be additional motivation for a structuralist account of scientific theories.

Finally, Michael Esfeld's paper focuses on the question of how to reconcile (ontic) structural realism with accounts of quantum non-locality. More particularly he responds to the worry that structural realism on its own cannot account for the spacelike separated, correlated measurement results in EPR experiments. Esfeld argues that structural realism needs to be complemented by primitive ontology theories of quantum mechanics, such as Bohmian mechanics or GRW theories. According to Esfeld it turns out that Bohmian Mechanics provides the best prospect for enabling structural realism to give a convincing account of quantum non-locality.

\section{References}

Ladyman, J. (2016). Structural realism. In E. N. Zalta (Ed.) The Stanford encyclopedia of philosophy (Winter 2016 Edition). https://plato.stanford.edu/archives/win2016/entries/structural-realism/. 
Ladyman, J., Ross, D., Spurret, D., \& Collier, J. (2007). Every thing must go: Metaphysics naturalised. Oxford: Oxford University Press.

Worrall, J. (1989). Structural realism: The best of both worlds? Dialectica, 43, 99-124. 